\title{
Influência da flora das florestas Amazônica e Atlântica na vegetação do cerrado sensu stricto
}

\author{
BEATRIZ B. MÉIO ${ }^{1}$, CRISTIANE V. FREITAS ${ }^{1}$, LEUSEROBERTA JATOBÁ $^{1}$, \\ MARIO E.F. SILVA ${ }^{1}$, JOSÉ F. RIBEIRO ${ }^{2}$ e RAIMUNDO P. B. HENRIQUES ${ }^{1,3}$
}

(recebido: 25 de julho de 2002; aceito: 14 de agosto de 2003)

\begin{abstract}
The influence of Amazonian and Atlantic flora in the vegetation of cerrado sensu stricto). The contribution of Amazonian and Atlantic floras was analyzed in twelve localities in the cerrado biome. A total of 290 tree and shrub species were recorded in those localities. Of these, $41.1 \%$ occurred only in cerrado and are probably endemic, and $58.9 \%$ also occurred in Atlantic and/or Amazonian forest. For non-endemic species the contribution of Atlantic forest was greater (44.8\%) than Amazonian ones (1.4\%), with the remaining $12.7 \%$ species found in both forest biomes. The proportions of species with centers of distribution in Atlantic and Amazonian forest showed a slight decrease toward the center of cerrado biome. For the former, the distance explained only $30 \%$ of variation in proportions by a polynomial model fit to the data, and for the latter a significant linear model explained 78\% of variations. The flora of Amazon forest and Atlantic forest showed a segregation with altitude. The proportions of species with centers of distribution in the Atlantic forest showed a slight increase with altitude, but a polynomial model explained only $18 \%$ of this variation. Inversely, there was a linear relationship between the proportions of species with centers of distribution in Amazonian forest and altitude that explained 31\% of variation. The differences in contribution patterns of the Atlantic and Amazonian forest flora to the cerrado physiognomy is discussed with respect to climatic changes during the Quaternary and to differences in plant species tolerance to fire and low temperature in the cerrado biome.
\end{abstract}

Key words - Amazon forest, Atlantic forest, cerrado, distribution patterns, phytogeography

RESUMO - (Influência da flora das florestas Amazônica e Atlântica na vegetação de cerrado sensu stricto). A contribuição das floras Atlântica e Amazônica foi analisada em doze localidades no bioma do cerrado. Um total de 290 espécies de arbustos e árvores foram registradas nas localidades de cerrado. Deste total $41,1 \%$ ocorreram apenas no cerrado, sendo provavelmente endêmicas e 58,9\% ocorreram nas florestas Atlântica e Amazônica. Para as espécies não endêmicas, a contribuição da floresta Atlântica foi maior (44,8\%) do que a da floresta Amazônica (1,4\%), com as 12,7\% de espécies restantes ocorrendo nos dois biomas florestais. A proporção de espécies com centro de distribuição na floresta Atlântica e Amazônica mostraram um pequeno decréscimo em direção ao centro do bioma do cerrado. Para o primeiro, a distância explicou apenas $30 \%$ da variação na proporção de espécies, por um modelo polinomial ajustado aos dados e, para o último, um modelo linear explicou 78\% da variação. A proporção de espécies com centro de distribuição na floresta Atlântica mostrou um pequeno aumento com a altitude, mas um modelo polinomial explicou apenas $18 \%$ dessa variação. Inversamente, ocorreu um decréscimo linear na proporção de espécies com a altitude para aquelas com centro de distribuição na floresta Amazônica; o modelo explicou 31\% da variação. As diferenças na contribuição das floras da floresta Atlântica e Amazônica para a fisionomia de cerrado é discutida com relação às mudanças climáticas durante o Quaternário e diferenças na tolerância entre espécies às queimadas e baixas temperaturas no bioma do Cerrado.

Palavras-chave - cerrado, floresta Amazônica, floresta Atlântica, fitogeografia, padrões de distribuição

\section{Introdução}

A dispersão e o intercâmbio de espécies entre biotas são dependentes de configurações geográficas e eventos históricos, os quais são importantes mecanismos reguladores da riqueza de espécies nas comunidades

\footnotetext{
1. Universidade de Brasília, Departamento de Ecologia, Caixa Postal 04457, 70919-970 Brasília, DF, Brasil.

2. Embrapa, Centro de Pesquisa Agropecuária dos Cerrados, Caixa Postal 08223, 73301-970 Planaltina, DF, Brasil.

3. Autor para correspondência: henriq@unb.br
}

(Schluter \& Ricklefs 1993a, b). Devido à sua localização, o bioma do cerrado se apresenta como uma região única para o estudo da influência que os fatores geográficos e históricos tiveram em sua biota. O bioma do cerrado se estende como um corredor xérico, conectando o chaco à caatinga, ocupando a região intermediária entre as duas maiores florestas úmidas neotropicais: a floresta Amazônica e a floresta Atlântica. A maior parte do bioma do cerrado apresenta um gradiente fisionômico com vegetação xeromorfa que varia de campo limpo até cerradão, embora também ocorram matas de galeria e florestas estacionais. As definições dos biomas da 
floresta Atlântica e Amazônica e do cerrado, incluindo uma descrição detalhada dos seus diferentes tipos fisionômicos de vegetação pode ser encontrada em Eiten (1972) e Oliveira Filho \& Ratter (2002).

Muitos estudos baseados em palinologia e distribuição atual de plantas têm apresentado substanciais evidências indicando que, em pelo menos três períodos nos últimos 60.000 anos AP, o clima da região do cerrado foi mais frio e seco do que no presente, ocorrendo a expansão das formações abertas sazonais e contração das florestas úmidas (Absy et al. 1991, Ledru 1993, Prado \& Gibbs 1993, Vicentini \& SalgadoLabouriau 1996). Segundo Salgado-Labouriau et al. (1997), o último período seco coincidiu com o meio para o fim do máximo glacial há 19.000-5.000 anos AP, após o qual a umidade voltou a aumentar coincidindo com a expansão das florestas úmidas no bioma do cerrado (Ledru 1993).

Estudos florísticos pioneiros mostraram que as florestas Amazônica e Atlântica influenciaram a flora do bioma do cerrado, sendo, no entanto maior a influência desta última (Smith 1962, Rizzini 1963). Uma análise quantitativa recentemente realizada por Oliveira Filho \& Fontes (2000), mostrou que a influência da floresta Atlântica na flora do cerrado ocorre através de uma conexão florística com as florestas estacionais do sudeste do Brasil.

A existência de padrões de distribuição disjunto em várias espécies de plantas da floresta Amazônica e Atlântica sugere também a possibilidade de conexão entre as floras destas florestas, no passado, através do bioma do cerrado (Rizzini 1963, Prance 1982). Vários estudos apóiam essa idéia, ressaltando a importância do papel das matas de galeria nessa conexão (Ratter et al. 1973, 1978, Mori et al. 1981, Prance 1987, Rizzini 1979). A esse respeito, os estudos de Oliveira Filho \& Ratter (1995a), mostraram que existe uma série de espécies Amazônicas e Atlânticas, que penetraram no bioma dos cerrados através das matas de galeria ou florestas estacionais.

Embora várias espécies de plantas possuam seu centro de distribuição nas florestas Amazônica ou Atlântica, pouco se conhece sobre o padrão geográfico dessa influência no bioma do cerrado. Os resultados do estudo de Oliveira Filho \& Fontes (2000), mostraram que a similaridade florística das florestas Atlântica com o bioma do cerrado, tende a diminuir com a distância para o interior do bioma; além disso foi detectada uma forte influência da temperatura e da altitude na diferenciação da vegetação na floresta Atlântica. Sobre esses temas foram propostas duas hipóteses: (1) a primeira propõe que a contribuição de organismos da floresta Amazônica e Atlântica diminui em direção ao centro do bioma dos cerrados (Cerqueira 1980, Silva \& Oniki 1988); (2) a segunda sugere que existe uma segregação altitudinal entre as espécies da floresta Amazônica e Atlântica, com as primeiras nas depressões periféricas e a segunda nos altos planaltos (Silva 1996).

É clara a importância que as matas de galeria tiveram na influência das florestas Amazônica e Atlântica sobre bioma do cerrado, servindo de corredores mésicos para os elementos dependentes de maior umidade nas floras dessas florestas (Oliveira Filho $\&$ Ratter 1995a). No entanto, pouco é conhecido sobre essa influência na fisionomia de cerrado sensu stricto. Neste trabalho, nós examinamos (1) qual a contribuição para a flora da fisionomia de cerrado sensu stricto das floras das florestas Atlântica e Amazônica, (2) se a proporção de espécies de plantas com centro de distribuição nas florestas úmidas (Amazônica e Atlântica), diminui com a distância da zona de contato em direção ao centro do bioma do cerrado, como proposto pela hipótese de Cerqueira (1980) e Silva \& Oniki (1988), e (3) se a proporção de espécies da floresta Atlântica e da floresta Amazônica segregam-se em diferentes altitudes no bioma dos cerrados como previsto pela hipótese de Silva (1996).

\section{Material e métodos}

Em virtude deste trabalho ter como principal objetivo a análise de padrões geográficos, da influência das florestas Amazônica e Atlântica na fisionomia de cerrado sensu stricto, foram escolhidas localidades atravessando o bioma dos cerrados no sentido noroeste - sudeste. Para um total de 12 localidades foram obtidas listas de espécies arbustivas e arbóreas da flora a partir da literatura e de levantamentos realizado por um dos autores (J.F. Ribeiro). As 12 localidades compreenderam apenas áreas em que a vegetação pode ser classificada fisionomicamente como cerrado (sensu stricto), baseado no tipo de habitat e na presença de espécies com ocorrência no cerrado (Eiten 1972, Ratter et al. 1996). Todas as localidades incluídas usaram parcelas, no entanto variaram no tamanho da amostra. A riqueza de espécies variou grandemente por localidade, sendo de 187 espécies na Chapada dos Guimarães (MT) e de 30 espécies em Pedro Leopoldo (MG). As informações para cada localidade são apresentadas na tabela 1 e mostradas na figura 1 .

Para a realização das análises utilizou-se uma lista florística, contendo informações sobre a presença e ausência das espécies em cada uma das 12 localidades. A lista não é apresentada aqui devido ao seu tamanho, mas está disponível mediante solicitação a um dos autores (R.P.B. Henriques). 
Tabela 1. Localidades, coordenadas, número de espécies, altitude e distância das localidades no bioma do cerrado da floresta Amazônica e da floresta Atlântica.

Table 1. Locality, coordinates, number of species, altitude and distance of localities in the cerrado biome from the Amazon forest and the Atlantic forest.

\begin{tabular}{|c|c|c|c|c|c|c|c|}
\hline Localidades & Lat. & Long. & $\begin{array}{l}\text { N. de } \\
\text { spécies }\end{array}$ & $\begin{array}{l}\text { Alt. } \\
\text { (m) }\end{array}$ & $\frac{\text { Distância da }}{\text { Amazônica }}$ & $\frac{\text { floresta (km }}{\text { Atlântica }}$ & Referências \\
\hline 1. Pedra Caída (MA) & $06^{\circ} 57^{\prime} \mathrm{S}$ & $47^{\circ} 28^{\prime} \mathrm{W}$ & 62 & 250 & 93 & 1019 & J.F. Ribeiro (dados não publicados) \\
\hline 2. Serra do Roncador (MT) & $12^{\circ} 49^{\prime} \mathrm{S}$ & $51^{\circ} 46^{\prime} \mathrm{W}$ & 129 & 400 & 240 & 981 & Ratter et al. (1973) \\
\hline 3. Chapada dos Guimarães (MT) & $15^{\circ} 21^{\prime} \mathrm{S}$ & $55^{\circ} 49^{\prime} \mathrm{W}$ & 188 & 380 & 130 & 1055 & Oliveira Filho \& Martins (1986) \\
\hline 4. Cuiabá (MT) & $15^{\circ} 36^{\prime} \mathrm{S}$ & $56^{\circ} 06^{\prime} \mathrm{W}$ & 37 & 190 & 130 & 1020 & Nascimento \& Saddi (1992) \\
\hline 5. Fazenda Água Limpa (DF) & $15^{\circ} 45^{\prime} \mathrm{S}$ & $47^{\circ} 57^{\prime} \mathrm{W}$ & 130 & 1120 & 555 & 500 & Ratter (1986) \\
\hline 6. Silvânia (GO) & $16^{\circ} 30^{\prime} \mathrm{S}$ & $48^{\circ} 30^{\prime} \mathrm{W}$ & 64 & 880 & 648 & 500 & Felfili \& Silva Junior (1993) \\
\hline 7. Coromandel (MG) & $18^{\circ} 20^{\prime} \mathrm{S}$ & $47^{\circ} 12^{\prime} \mathrm{W}$ & 43 & 880 & 833 & 370 & Brandão \& Gavilanes (1992) \\
\hline 8. Pedro Leopoldo (MG) & $19^{\circ} 39^{\prime} \mathrm{S}$ & $44^{\circ} 03^{\prime} \mathrm{W}$ & 28 & 698 & 1055 & 111 & Rizzini (1975) \\
\hline 9. Araxá (MG) & $19^{\circ} 46^{\prime} \mathrm{S}$ & $46^{\circ} 55^{\prime} \mathrm{W}$ & 39 & 1200 & 963 & 222 & Brandão \& Gavilanes (1992) \\
\hline 10. Emas (SP) & $22^{\circ} 02^{\prime} \mathrm{S}$ & $47^{\circ} 30^{\prime} \mathrm{W}$ & 33 & 730 & 1018 & 148 & Ferri \& Coutinho (1958) \\
\hline 11. Itirapina (SP) & $22^{\circ} 08^{\prime} \mathrm{S}$ & $47^{\circ} 47^{\prime} \mathrm{W}$ & 43 & 730 & 1111 & 92 & Durigan et al. (1994) \\
\hline 12. Botucatu (SP) & $22^{\circ} 45^{\prime} \mathrm{S}$ & $48^{\circ} 25^{\prime} \mathrm{W}$ & 53 & 550 & 1166 & $\begin{array}{l}\mathrm{Si} \\
(1\end{array}$ & $\begin{array}{l}\text { Silberbauer-Gottsberger \& Eiten } \\
\text { (1983) }\end{array}$ \\
\hline
\end{tabular}

Antes da realização das análises, a lista com 320 espécies foi revisada para confirmação dos nomes e sinônimos, resultando em 290 espécies.

Para a determinação da proporção de espécies oriundas da floresta Amazônica sensu lato e floresta Atlântica sensu stricto (Oliveira Filho \& Fontes 2000), foi registrada a ocorrência das 290 espécies das 12 localidades do bioma cerrado, nestes dois biomas. No conceito de floresta Atlântica s. str. são incluídas apenas as formações costeiras até $300 \mathrm{~km}$ para o interior, onde a precipitação é decorrente das massas de ar oceânicas que se condensam nas encostas montanhosas costeiras (Hueck 1972, Oliveira Filho \& Fontes 2000).

O registro das espécies foi preparado utilizando as listas de Heringer et al. (1977), Mendonça et al. (1998), Oliveira Filho \& Ratter (1995 b), Rizzini (1963), o banco de dados disponibilizado na Internet por Siqueira et al. (1997) além do banco de dados de Oliveira Filho \& Fontes (2000). Com essas informações, foi calculado, para cada localidade do bioma do cerrado a proporção de espécies com ocorrência na floresta Amazônica e Atlântica. Na análise da variação geográfica destas proporções, mediu-se a distância de cada localidade para o limite mais próximo de cada um desses dois biomas, usando um mapa com escala 1:5.000.000 do IBGE (1988).

O padrão geográfico de variação das proporções (p) de espécies da floresta Atlântica e Amazônica foi analisado usando ajustes lineares e não lineares. Os dados de proporções foram transformados em arcoseno $(\sqrt{ } \mathrm{p})$ para as análises estatísticas. Para cada caso, foi escolhido o ajuste que apresentasse o maior coeficiente de determinação $\left(\mathrm{r}^{2}\right)$. $\mathrm{Na}$ análise do efeito da altitude foram consideradas apenas



Figura 1. Mapa mostrando os locais de estudo na área core do bioma do cerrado. A linha contínua indica os limites dos biomas da floresta Amazônica e floresta Atlântica. Os números referem-se às localidades descritas na tabela 1 .

Figure 1. Map showing the localities of study in the core area of cerrado biome. The continuous line shows the limits of the Amazon forest and the Atlantic forest biomes. The numbers are the localities from table 1 . 
as localidades que estavam a menos $1.000 \mathrm{~km}$ do bioma da Floresta Amazônica, pois além desse limite não foi mais detectada a presença de espécies desse bioma.

\section{Resultados}

Do total de espécies compiladas (290), 119 espécies $(41,1 \%)$ ocorreram apenas nas localidades com cerrado sensu stricto, sendo provavelmente endêmicas do bioma do cerrado. As espécies não endêmicas corresponderam a $171(58,9 \%)$ com ocorrência nas florestas Atlântica e/ ou Amazônica. Para as espécies não endêmicas do cerrado à proporção daquelas com ocorrência apenas na floresta Atlântica, foi muito maior $(130 ; 44,8 \%)$ do que as com ocorrência na Floresta Amazônica ( $4 ; 1,4 \%)$, o restante $(37 ; 12,8 \%)$ ocorreu nos dois biomas florestais.

Um ajuste polinomial apresentou a maior explicação (32\%) para a relação entre a proporção de espécies da floresta Atlântica e a distância para o centro do bioma do cerrado (figura 2A). Essa relação mostrou um pequeno incremento a $600 \mathrm{~km}$ da floresta Atlântica, com posterior declínio para o centro do bioma do cerrado. A variação na proporção de espécies da floresta Amazônica com a distância desse bioma mostrou uma correlação linear significativa $(r=-0,88 ; p<0,05)$, com diminuição na proporção de espécies da Amazônia até uma distância de aproximadamente $1.000 \mathrm{~km}$ do contato com este bioma.

A relação da proporção de espécies da floresta Atlântica e Amazônica com a altitude dentro do bioma do cerrado é mostrada na figura $2 \mathrm{~B}$. A relação da proporção de espécies da floresta Atlântica com a altitude foi descrita por uma equação polinomial de segunda ordem, mas a explicação do modelo foi de apenas $18 \%$. Os resultados desta análise sugerem um pequeno aumento na proporção de espécies da floresta Atlântica com a altitude. Para as espécies da floresta Amazônica, a correlação foi negativa com a altitude, indicando uma diminuição na proporção de espécies, mas a correlação não foi significativa $(r=-0,56 ; p>0,05)$.

\section{Discussão}

O valor de endemismo encontrado neste estudo (41\%), para as espécies de cerrado sensu stricto é semelhante ao valor de 43,4\% encontrado por Heringer et al. (1977) em sua análise para a flora de todo o bioma do cerrado. Estes valores são inferiores ao valor de endemismo para espécies arbóreas (53,3\%), encontrado para a floresta Atlântica por Mori et al. (1981). O nível de endemismo da fisionomia de cerrado foi maior do que o valor de $12,2 \%$ que foi registrado para as matas de galeria na Chapada dos Guimarães (MT) por Pinto \& Oliveira Filho (1999).

Como observado neste estudo, existe uma grande proporção de espécies oriundas da floresta Atlântica $(44,8 \%)$ e uma proporção bem menor da floresta Amazônica $(1,4 \%)$. Na sua análise florística das
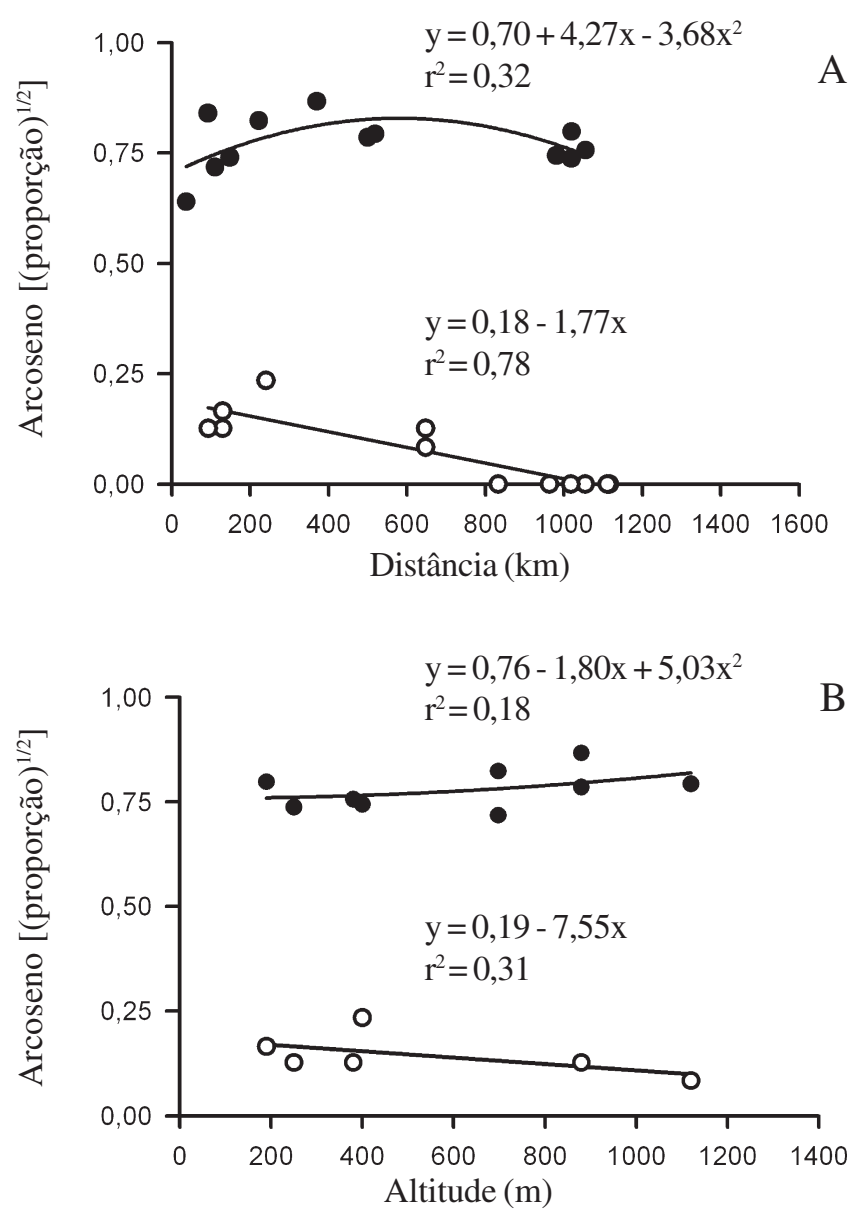

Figura 2. Variação na proporção de espécies com a altitude e a distância dos centros de distribuição nas florestas Amazônica e Atlântica no bioma dos cerrado. (A) Proporção

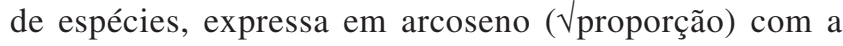
distância da floresta Atlântica $(\bullet)$ e da floresta Amazônica (O) no bioma do cerrado; (B) Proporção de espécies expressa,

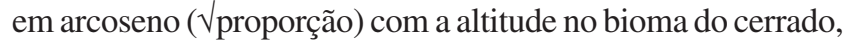
para as espécies com centro de distribuição na floresta Atlântica $(\bullet)$ e na floresta Amazônica (O).

Figure 2. Variation in the proportion of species with altitude and distance from center of distribution the Amazon and Atlantic forests in the cerrado biome. (A) Proportion of species in arcosin ( $\sqrt{\text { proportion}) ~ w i t h ~ d i s t a n c e ~ f r o m ~ A t l a n t i c ~}$ Forest (•) and Amazon Forest (O) in the cerrado biome; (B)

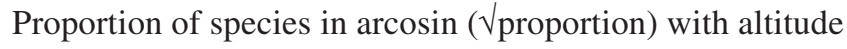
in the cerrado biome, for species with center of distribution in the Atlantic forest $(\bullet)$ and in the Amazon forest (O). 
espécies lenhosa para todo o bioma dos cerrados, Rizzini (1963) encontrou o valor de $26 \%$ para a proporção de espécies de ocorrência na floresta Atlântica e 12\% para as da floresta Amazônica. Em estudo recente, Oliveira Filho \& Fontes (2000) apresentaram resultados semelhantes, mostrando que a flora arbórea do bioma do cerrado tem maior afinidade com a floresta Atlântica (55\% de espécies comuns) do que com a floresta Amazônica (20\%). Entre os dois tipos de formação florestal reconhecido por esses autores para a floresta Atlântica, a floresta estacional (semidecídua) apresenta uma afinidade florística maior com o bioma do cerrado do que a floresta ombrófila. Todos estes resultados indicam a existência de uma maior afinidade florística do bioma do cerrado com a floresta Atlântica.

Oliveira Filho \& Ratter (1995a) apresentam dados mostrando que, para as matas de galeria, a proporção de espécies com ocorrência na floresta Amazônica é menor (8\%-19\%) do que as de ocorrência na floresta Atlântica (30\%-40\%). Pinto \& Oliveira Filho (1999) encontraram, em mata de galeria na Chapada dos Guimarães, valores semelhantes para a proporção de espécies da floresta Atlântica e da floresta Amazônica (30\%). A proporção de espécies nos cerradões, com ocorrência na floresta Atlântica foi de $25 \%$ e para as com ocorrência na floresta Amazônica 8\% (Oliveira Filho \& Ratter 1995a). Esses estudos sugerem que a influência florística da floresta Atlântica e Amazônica no bioma do cerrado pode variar com a localização geográfica e o tipo de fitofisionomia. Entre os tipos fisionômicos do bioma do cerrado, a mata de galeria parece ser mais favorável para as espécies oriundas da floresta Amazônica do que as fisionomias mais abertas dos cerrados (cerrado s. str. e cerradão), pois enquanto para as matas de galeria a proporção de espécies com distribuição nesse bioma variaram de 8\% a 30\% (Oliveira Filho \& Ratter 1995a, Pinto \& Oliveira Filho 1999), para as fisionomias mais abertas variaram de $1,4 \%$ a $8 \%$ (Oliveira Filho \& Ratter 1995a, este estudo).

O resultado da análise da variação das proporções de espécies com a distância da floresta Atlântica e Amazônica foi consistente com a hipótese de diminuição em direção ao centro do bioma do cerrado (Cerqueira 1980, Silva \& Oniki 1988). Para a floresta Amazônica, uma correlação linear com a distância foi significativa $(\mathrm{p}<0,05)$ e explicou uma grande parte na diminuição na proporção de espécies (78\%) desse bioma no cerrado. Em função dessa diminuição, as espécies da floresta Amazônica penetram menos no bioma dos cerrados, alcançando no máximo, $700 \mathrm{~km}$ da floresta Amazônica, em Silvânia (MG) (figura 1).
A menor contribuição da flora da floresta Amazônica, em comparação com a da floresta Atlântica, pode ser atribuída às diferenças na história dessas duas regiões. Como sugerido por Silva (1996), grande parte da zona de contato do bioma do cerrado com a floresta Amazônica apresenta uma depressão geomorfológica com altitude variando de 100 a $500 \mathrm{~m}$, sendo possível que, durante os períodos mais secos do Quaternário (Salgado-Labouriau et al. 1997), essa conexão tenha sofrido mudanças drásticas (Ab’Saber 1977, 1983, 1988) com efeitos negativos na colonização do bioma do cerrado por espécies da floresta Amazônica. Diferentemente, a região de conexão do bioma cerrado com a floresta Atlântica parece ter sido menos influenciada pelas mudanças climáticas do Quaternário (Brown \& Ab'Saber 1979). Portanto, as mudanças climáticas do Quaternário podem ter tido um drástico efeito na distribuição das espécies da floresta Amazônica em comparação com as espécies da floresta Atlântica no bioma do cerrado.

Outro fator que pode estar também limitando a distribuição das espécies da floresta Amazônica no bioma do cerrado é o clima. $\mathrm{O}$ afastamento da zona de contato do bioma dos cerrados - floresta Amazônica coincide com o aumento da latitude e diminuição da temperatura mínima de inverno (Nimer 1979). Paralelamente, ocorre também um aumento da estação seca da Amazônia para a região do cerrado (Salati 1985). É possível que as espécies da floresta Amazônica sejam mais sensíveis ao clima mais frio e seco do cerrado, o que, juntamente com um solo muito pobre em nutrientes (Adámoli et al. 1987), poderia explicar a menor ocorrência e distribuição de espécies da floresta Amazônica no bioma do cerrado, particularmente na fisionomia de cerrado sensu estricto. Por outro lado o clima da floresta Atlântica parece ser mais similar ao do bioma do cerrado, com ocorrência também de baixas temperaturas no inverno e estação seca pronunciada (Nimer 1979). Nesta categoria se enquadra a floresta Atlântica do tipo ombrófila, que ocorre acima de $700 \mathrm{~m}$ de altitude da região sudeste do Brasil (Oliveira Filho \& Fontes 2000). Tal similaridade poderia explicar a maior proporção e distribuição de espécies arbustivo - arbórea da floresta Atlântica no bioma do cerrado.

O fogo é outro fator que pode explicar, em parte, a menor proporção e distribuição de espécies da floresta Amazônica no bioma do cerrado. As queimadas são freqüentes no bioma do cerrado, onde podem ocorrer em média a cada dois anos (Eiten 1972). Considerando que o fogo pode ser letal para aproximadamente $98 \%$ dos arbustos e árvores da floresta Amazônica (Uhl \& 
Kauffman 1990), é de se esperar uma baixa ocorrência de espécies deste bioma no bioma do cerrado onde a frequiência de queimadas é maior. Infelizmente não existem resultados comparáveis para as espécies da floresta Atlântica. No entanto, podemos ter uma idéia do desempenho das plantas desse bioma ao fogo, através da sua capacidade de se reproduzir vegetativamente, uma vez que esse caráter tem sido associado à resistência das plantas às queimadas (Lacey et al. 1982, Frost 1984). Informações circunstanciais indicam que as plantas da floresta Amazônica apresentam pequena capacidade de se reproduzir vegetativamente após as queimadas (Uhl et al. 1981), comparativamente às plantas da floresta Atlântica (Castellani \& Stubblebine 1993).

As espécies da floresta Atlântica e Amazônica deste estudo ocorreram de $200 \mathrm{~m}$ até $1.260 \mathrm{~m}$ de altitude. $\mathrm{O}$ principal fator que varia com a altitude é a temperatura, que apresenta diminuição adiabática de $-1{ }^{\circ} \mathrm{C}$ a cada $100 \mathrm{~m}$ de altitude (Rosenberg 1974). Segundo a hipótese de Silva (1996), seria de se esperar uma maior proporção de espécies da floresta Atlântica em altitudes maiores no bioma do cerrado, já que sua flora esta aclimatada a temperaturas mais baixas. Para as espécies da floresta Amazônica deveria ser encontrado o inverso desse padrão. Os resultados deste estudo são consistentes com esta hipótese, no entanto, a variação com a altitude foi pequena para uma conclusão definitiva.

Agradecimentos - Os autores desejam agradecer a Ary Teixeira de Oliveira Filho pelo fornecimento das suas planilhas de espécies. A James Alexander Ratter pela leitura crítica do manuscrito, correções e sugestões. Aos dois revisores anônimos pelas críticas e sugestões ao manuscrito. A Reginaldo Constantino pela confecção do mapa do trabalho.

\section{Referências bibliográficas}

AB'SABER, A.N. 1977. Os domínios morfoclimáticos da América do Sul. Primeira aproximação. Geomorfologia 52:1-21.

AB'SABER, A.N. 1983. O domínio dos cerrados: Introdução ao conhecimento. Revista do Servidor 111:41-55.

AB'SABER, A.N. 1988. O pantanal mato-grossense e a teoria dos refúgios. Revista Brasileira de Geografia 50:9-57.

ABSY, M.L., CLEEF, A., FOURNIER, M., MARTIN, L., SERVANT, M., SIFEDDINE, A., SILVA, M.F., SOUBIES, F., SUGUIO, K., TURCQ, B. \& VAN DER HAMMEN, T. 1991. Mise en évidence de quatre phase dóuverture de la forêt dense dans le sud-est de l'Amazonie au cours des 60.000 dernières années. Première comparison avec dáutres régions tropicales. Comptes Rendus Academie des Sciences, Paris 312:673-678.
ADÁMOLI, J., MACÊDO, J., AZEVEDO, L.G. \& NETTO, J. M. 1987. Caracterização da região dos cerrados In Solos dos cerrados. Tecnologias e estratégias de manejo (W.J. Goedert, ed.). Embrapa/ CPAC, Planaltina, p.33-74.

BRANDÃO, M. \& GAVILANES, M.L. 1992. Espécies arbóreas padronizados do cerrado mineiro e sua distribuição no Estado. Informação Agropecuária 16:5-11.

BROWN, K.S. \& AB'SABER, A.N. 1979. Ice-age refuges and evolution in the Neotropics: correlation of paleoclimatological, geomorphological and pedological data with modern biological endemism. Paleoclimas 5:1-30.

CASTELLANI, T.T. \& STUBBLEBINE, W.H. 1993. Sucessão secundária em mata tropical mesófila, após perturbação por fogo. Revista Brasileira de Botânica 16:181-203.

CERQUEIRA, R. 1980. South American landscapes and their mammals. In Mammalian biology in South America (M.A. Mares \& H.H. Genoways, eds.). Special Publication Series, Pymatuning Laboratory of Ecology, University of Pittsburg 6:53-75.

DURIGAN, G.H., LEITÃO FILHO, H.F. \& RODRIGUES, R.R. 1994. Phytosociology and structure of a frequently burnt cerrado vegetation in SE Brazil. Flora 189:153-160.

EITEN, G. 1972. The cerrado vegetation of Brazil. Botanical Review 38:201-341.

FELFILI, J.M. \& SILVA JUNIOR, M.C. 1993. A comparative study of cerrado (sensu stricto) vegetation in Central Brazil. Journal of Tropical Ecology 9:277-289.

FERRI, M.G. \& COUTINHO, L.M. 1958. Contribuição ao conhecimento da ecologia do cerrado. Estudo comparativo da economia d'água de sua vegetação, em Emas (Est. de São Paulo), Campo Grande (Est. de Mato Grosso) e Goiânia (Est. de Goiás). Boletim da Faculdade de Filosofia Ciências e Letras da USP, Botânica 15:103-150.

FROST, P.G.H. 1984. The responses and survival of organisms in fire-prone environments. In Ecological effects of fire in South African ecosystems (P.V. Booysen \& N.M. Tainton, eds.). Springer-Verlag, Berlin, p.273-310.

HERINGER, E.P., BARROSO, G.M., RIZZO, J.A. \& RIZZINI, C.T. 1977. A flora do cerrado. In IV Simpósio sobre o cerrado: Bases para a utilização agropecuária (M.G. Ferri, org.). Edusp, São Paulo, p.211-232.

HUECK, K. 1972. As florestas da América do Sul. Ecologia, composição e importância econômica. Editora da Universidade de Brasília \& Editora Polígono, São Paulo.

IBGE. 1988. Mapa de vegetação do Brasil. Escala 1:5.000.000, IBGE, Rio de Janeiro.

LACEY, C., WALKER, J. \& NOBLE, I. R. 1982. Fire in autralian tropical savannas. In Ecology of tropical savannas (B.J. Huntley \& B.H. Walker, eds.). Springer-Verlag, Berlin, p.246-272.

LEDRU, M. 1993. Late quaternary environmental and climatic changes in central Brazil. Quaternary Research 39:90-98. 
MENDONÇA, R.C., FELFILI, J.M., WATER, B.M.T., SILVA JUNIOR, M.C., REZENDE, A.V., FILGUEIRAS, T.S. \& NOGUEIRA, P.E. 1998. Flora vascular do cerrado. In Cerrado: Ambiente e flora. (S.M. Sano \& S.P. Almeida, eds.). Embrapa, Planaltina, p.289-556.

MORI, S.A., BOOM, B.M. \& PRANCE, G.T. 1981. Distribution patterns and observation of eastern Brazilian coastal forest tree species. Brittonia 33:233-245.

NASCIMENTO, M.T. \& SADDI, N. 1992. Structure and floristic composition in an area of cerrado in Cuiabá MT , Brasil. Revista Brasileira de Botânica 15:47-55.

NIMER, E. 1979. Climatologia do Brasil. IBGE, Rio de Janeiro.

OLIVEIRAFILHO, A.T. \& FONTES, M.A.L. 2000. Patterns of floristic differentiation among Atlantic forests in Southeastern Brazil and the influence of climate. Biotropica 32:793-810.

OLIVEIRAFILHO, A.T. \& MARTINS, F.R. 1986. Distribuição, caracterização e composição florística das formações vegetais da região da Salgadeira, na Chapada dos Guimarães (MT). Revista Brasileira de Botância 9:207-223.

OLIVEIRA FILHO, A.T. \& RATTER, J.A. 1995a. A study of the origin of central Brazilian forests by the analysis of plant species distribution patterns. Edinburgh Journal of Botany 52:141-194.

OLIVEIRA FILHO, A.T. \& RATTER, J.A. 1995b. Database, woody flora of 106 forest areas of eastern tropical South America. Occasional Monographs, Royal Botanic Garden Edinburgh, Edinburgh.

OLIVEIRA FILHO, A.T. \& RATTER, J.A. 2002. Vegetation physiognomies and woody flora of the cerrado biome. In The cerrados of Brazil. Ecology an natural history of a Neotropical savanna (P.S. Oliveira \& R.J. Marquis, eds.). Columbia University Press, New York, p.91-120.

PINTO, J.R.R. \& OLIVEIRAFILHO, A.T. 1999. Perfil florístico e estrutura da comunidade arbórea de uma floresta de vale no Parque Nacional da Chapada dos Guimarães, Mato Grosso, Brasil. Revista Brasileira de Botânica 22:53-67.

PRADO, D.E. \& GIBBS, P.E. 1993. Patterns of species distributions in the dry seasonal forests of South America. Annals of Missouri Botanical Garden 80:902-927.

PRANCE, G.T. 1982. Forest refuges: Evidence from woody Angiosperms. In Biological diversification in the tropics (G.T. Prance, ed.). Columbia University Press, New York, p.137-156.

PRANCE, G.T. 1987. Biogeography of neotropical plants. In Biogeography and quaternary history in tropical America (T.C. Whitmore \& G.T. Prance, eds.). Claredon Press, Oxford, p.46-65.

RATTER, J.A. 1986. Notas sobre a vegetação da Fazenda Água Limpa (Brasília, DF, Brasil). Textos Universitários n. 3, Editora UnB, Brasília.
RATTER, J.A., RICHARD, P.W., ARGENT, GT. \& GIFFORD, D.R. 1973. Observations on the vegetation of northeasthern Mato Grosso. I. The woody vegetation types of the Xavantina-Cachimbo Expedition area. Philosophical Transections of the Royal Society of London B 266:44-492.

RATTER, J.A., ASKEW, G.P., MONTGOMERY, R.F. \& GIFFORD, D.R. 1978.Observations on the vegetation of northeastern Mato Grosso. II. Forests and soils of the Rio Suiá-Missu area. Proceedings of Royal Society of London B 203:191-208.

RATTER, J.A., BRIDGEWATER, S., ATKINSON, R. \& RIBEIRO, J.F. 1996. Analysis of the florístic composition of the brazilian cerrado vegetation. II. Comparison of the woody vegetation of 98 areas. Edingurgh Journal of Botany 53:153-180.

RIZZINI, C.T. 1963. A flora do cerrado. Análise florística das savannas centrais. In Simpósio sobre o cerrado (M.G. Ferri, org.). Edusp, São Paulo, p.126-177.

RIZZINI, C.T. 1975. Contribuição ao conhecimento da estrutura do cerrado. Brasil Florestal 22:3-15.

RIZZINI, C.T. 1979.Tratado de fitogeografia do Brasil. Aspectos sociológicos e florísticos. Hucitec-Edusp, São Paulo, v.2.

ROSENBERG, N.J. 1974. Microclimate: The biological environment. John Wiley \& Sons, New York.

SALGADO-LABOURIAU, M.L., BARBERI, M., VICENTINI, K.R.F. \& PARIZZI, M.G. 1997. A dry climatic event during the late quaternary of tropical Brazil. Review of Palaeobotany and Palynology 99:115-129.

SALATI, E. 1985. A floresta e as águas. Ciência Hoje 3:58-64. SCHLUTER, D. \& RICKLEFS, R.E. 1993a. Species diversity: an introduction to the problem. In Species diversity in ecological communities (R.E. Ricklefs \& D. Schuter, eds.). University of Chicago, Chicago, p.1-10.

SCHLUTER, D. \& RICKLEFS, R.E. 1993b. Species diversity: regional and historical influences. In Species diversity in ecological communities (R.E. Ricklefs \& D. Schuter, eds.). University of Chicago, Chicago, p.350-364.

SILBERBAUER-GOTTSBERGER, I. \& EITEN, G. 1983. Fitossociologia de um hectare de cerrado. Brasil Florestal 54:55-70.

SILVA, J.M.C. 1996. Distribution of Amazonian and Atlantic birds in gallery forests of the cerrado region, South America. Ornitologia Neotropical 7:1-18.

SILVA, J.M.C. \& ONIKI, Y. 1988. Lista preliminar da avifauna da Estação Ecológica Serra das Araras, Mato Grosso, Brasil. Boletim do Museu Paraense Emílio Goeldi, ser. Zoologia 4:123-143.

SMITH, L.B. 1962. Origins of the flora of southern Brazil. Contributions from the United States National Herbarium 35:215-249. 
SIQUEIRA, M. F., MARTINS, F.R. \& TAMASHIRO, J. 1997. Espécies arbóreas da mata atlântica (www.bdt.org.br/ mata.atlantica/flora).

UHL, C \& KAUFFMAN, J.B. 1990. Deforestation, fire susceptibility, and potential tree responses to fire in the eastern Amazon. Ecology 71:437-449.
UHL, C., CLARK, K., CLARK, H. \& MURPHY, P. 1981. Early plant succession after cutting and burning in the upper Rio Negro region of the Amazon Basin. Journal of Ecology 69:631-649.

VICENTINI, K.R.F. \& SALGADO-LABOURIAU, M.L. 1996. Palynological analysis of a palm swamp in central Brazil. Journal of South American Earth Sciences 9:207-219. 